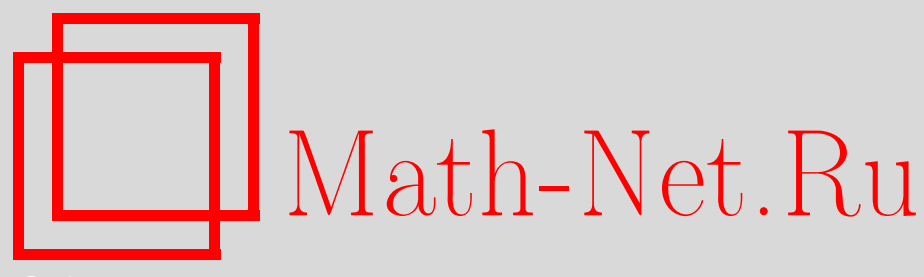

Д. Ю. Письмак, Ю. М. Письмак, Моделирование взаимодействия материальной плоскости со спинорным полем в рамках подхода Симанзика, ТМФ, 2015, том 184, номер 3, 505-519

DOI: https://doi.org/10.4213/tmf8922

Использование Общероссийского математического портала Math-Net.Ru подразумевает, что вы прочитали и согласны с пользовательским соглашением http: //www . mathnet.ru/rus/agreement

Параметры загрузки:

IP : 34.227 .88 .159

26 апреля 2023 г., 16:53:53

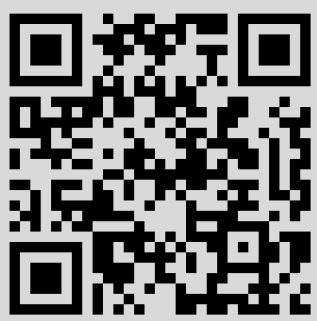


Том 184, № 3

сентябрь, 2015

(C) 2015 г. $\quad$ Д. Ю. Письмак*, Ю. М. Письмак*

\title{
МОДЕЛИРОВАНИЕ ВЗАИМОДЕЙСТВИЯ МАТЕРИАЛЬНОЙ ПЛОСКОСТИ СО СПИНОРНЫМ ПОЛЕМ В РАМКАХ ПОДХОДА СИМАНЗИКА
}

\begin{abstract}
Предлагается модель взаимодействия спинорного поля с материальной плоскостью, построенная на основе подхода Симанзика. Исследованы процессы рассеяния дираковских частиц на плоскости и свойства локализованных вблизи нее состояний. Модель может найти применение для широкого класса явлений, возникающих при взаимодействии полей квантовой электродинамики с двумерными материалами.
\end{abstract}

Ключевые слова: эффект Казимира, электродинамика в пространстве с макроскопическими неоднородностями, потенциал Черна-Саймонса, нанофизика, взаимодействие дираковских полей с двумерными объектами.

DOI: $10.4213 / \operatorname{tmf} 8922$

\section{1. ВВЕДЕНИЕ}

Квантово-полевые модели элементарных частиц обычно рассматриваются в однородном и изотропном пространстве-времени [1]. Это вполне естественно при изучении различных процессов с простейшими возбуждениями вакуума. Однако, если свойства вакуума существенно меняются в результате взаимодействий квантовых полей с макроскопическими объектами, такой подход неприменим. В этом случае в динамике материальных тел могут возникать квантовые макроэффекты, не объяснимые в рамках классической физики.

Теоретически эта проблема была впервые рассмотрена в 1948 году Казимиром [2]. Он показал, что вследствие флуктуаций квантового вакуума возникает притяжение между двумя идеально проводящими пластинами плоского незаряженного конденсатора. Это явление, которое получило название эффекта Казимира (ЭК), наблюдается экспериментально, и полученные для хорошо проводящих материалов эмпирические результаты с высокой степенью точности согласуются с теоретическими [3]-[6].

${ }^{*}$ Санкт-Петербургский государственный университет, Санкт-Петербург, Россия. E-mail: dashap2@gmail.com, ypismak@yahoo.com 
На характерных для ЭК расстояниях 10-1000 нм как классические, так и квантовые свойства системы оказываются существенными, что формирует особую нанофизику, исследование которой представляет не только теоретический интерес. Понимание ее закономерностей важно также для разработки новых технических устройств из-за все возрастающей тенденции к их миниатюризации.

Хотя теоретическим исследованиям ЭК посвящено много работ [6], [7], однако в них часто используются упрощенные модели свободной скалярной теории поля или свободного электромагнитного поля с фиксированными граничными условиями. Они применимы для исследования только отдельных аспектов ЭК, и многие важные особенности квантовой электродинамики в них не учитываются. Такие модели непригодны для полного описания широкого круга нанофизических явлений, возникающих в системе в результате взаимодействия ее квантовых степеней свободы с материальными телами.

Основой представленных в настоящей работе исследований служит подход Симанзика [8], в котором взаимодействия квантованных полей с пространственной неоднородностью (дефектами) моделируются дополнительным функционалом действия (действием дефекта), сосредоточенным в той области пространства, где эта неоднородность - макроскопический объект - находится. Важным предположением является также выполнение обычных требований, предъявляемых к квантовополевым моделям (локальность, перенормируемость, симметрийные свойства).

В связи с последними достижениями в области физики двумерных материалов особое внимание уделяется квантово-полевым моделям с двумерными пространственными неоднородностями. В квантовой электродинамике (КЭД) взаимодействие фотонов с двумерным дефектом полностью определяется требованиями локальности, калибровочной инвариантности, перенормируемости и описывается функционалом действия Черна-Саймонса с одной безразмерной константой, характеризующей свойства материала [9]. От нее зависит сила Казимира, которая оказывается тем самым неуниверсальной и для плоского конденсатора может быть не только притягивающей, но и отталкивающей [9].

В рамках этой модели было также показано, что при взаимодействии плоского дефекта с электрическим зарядом возникает магнитное поле, а постоянный ток, параллельный плоскости, генерирует электрическое поле [9]. Расчеты потенциала Казимира-Полдера для нейтрального атома вблизи плоской поверхности позволили найти нарушающую четность поправку к ранее известным результатам [10].

Взаимодействие электромагнитных волн с двумерными плоскими дефектами в модели с потенциалом Черна-Саймонса исследовалось в работах [11], [12], где было показано, что взаимодействие Черна-Саймонса не меняет закон Снеллиуса, однако меняет коэффициенты прохождения и отражения, которые зависят от величины константы связи. Кроме того, оно приводит к перемешиванию параллельных и перпендикулярных компонент поляризации (ТЕ- и ТМ-мод) электромагнитных волн и изменяет соотношение между частотой и волновым вектором для волн, распространяющихся в промежутке между двумя полностью отражающими средами. Это дает возможность, в частности, определить оптическими методами константу взаимодействия Черна-Саймонса, через которую выражается холловская проводимость. 
При конечной константе потенциал Черна-Саймонса нарушает пространственную четность и инвариантность относительно обращения времени. Это имеет место также в модели взаимодействия фотонов с полем Дирака размерности $2+1$, используемой для исследования свойств двумерных материалов [13], [14]. В этой модели не представлены реальные дираковские поля в $(3+1)$-мерном пространстве-времени, поэтому она пригодна для описания процессов с электронами и позитронами только внутри материальной плоскости, что существенно ограничивает область применения модели.

В настоящей работе для моделирования взаимодействия двумерного материального объекта с дираковским полем предлагается использовать подход Симанзика в обычной КЭД в $(3+1)$-мерном пространстве-времени. Подобная задача для спинорного поля рассматривалась в работах [15]. В качестве функционала действия дефекта, кроме фотонного действия Черна-Саймонса, требуется учесть чисто дираковский вклад, соответствующий базисным принципам КЭД. В рамках этой модели мы исследуем процессы рассеяния спинорных частиц на материальной плоскости, а также локализованные в ее окрестности связанные состояния.

\section{2. ПОСТАНОВКА ЗАДАЧИ}

Для описания взаимодействия квантового поля с материальным объектом (дефектом) Симанзик предложил использовать функционал действия вида

$$
S(\varphi)=S_{V}(\varphi)+S_{\mathrm{def}}(\varphi)
$$

Здесь $S_{V}$ - действие исходной квантово-полевой системы, $S_{\mathrm{def}}-$ действие дефекта:

$$
S_{V}(\varphi)=\int L(\varphi(x)) d^{D} x, \quad S_{\mathrm{def}}(\varphi)=\int_{\Gamma} L_{\mathrm{def}}(\varphi(x)) d^{D^{\prime}} x
$$

где $\Gamma$ - подпространство размерности $D^{\prime} \leqslant D$ в $D$-мерном пространстве [8]. Основные принципы КЭД - калибровочная инвариантность, локальность, перенормируемость - налагают сильные ограничения на возможный вид действия дефекта $S_{\text {def }}$. Взаимодействие с электромагнитным полем $A_{\mu}(x)$ двумерной поверхности без зарядов и токов, форма которой определяется уравнением $\Phi(x)=0, x=\left(x_{0}, x_{1}, x_{2}, x_{3}\right)$, описывается функционалом Черна-Саймонса:

$$
S_{\mathrm{def}}(A)=\frac{a}{2} \int \varepsilon^{\lambda \mu \nu \rho} \partial_{\lambda} \Phi(x) A_{\mu}(x) F_{\nu \rho}(x) \delta(\Phi(x)) d^{4} x
$$

где $F_{\nu \rho}(x)=\partial_{\nu} A_{\rho}-\partial_{\rho} A_{\nu}, \varepsilon^{\lambda \mu \nu \rho}$ обозначает полностью антисимметричный тензор $\left(\varepsilon^{0123}=1\right)$, параметр $a-$ безразмерная константа взаимодействия. Выражение представляет собой наиболее общую форму сосредоточенного на поверхности дефекта калибровочно-инвариантного действия, инвариантного относительно перепараметризации и не содержащего параметров отрицательной размерности. 
Фермионный вклад в действие дефекта в самом общем виде записывается следующим образом:

$$
S_{\mathrm{def}}(\bar{\psi}, \psi)=\sum_{j=1}^{16} \int \alpha_{j} \bar{\psi}(x) \Gamma_{j} \psi(x) \delta(\Phi(x)) d x
$$

где $\Gamma_{j}-16$ базисных матриц Дирака, $\alpha_{j}$ - безразмерные константы взаимодействия. Полное действие модели, удовлетворяющее требованиям локальности, калибровочной инвариантности и перенормируемости, имеет вид

$$
S(\bar{\psi}, \psi, A)=-\frac{1}{4} F_{\mu \nu} F^{\mu \nu}+\bar{\psi}(i \hat{\partial}-m+i e \hat{A}) \psi+S_{\mathrm{def}}(A)+S_{\mathrm{def}}(\bar{\psi}, \psi)
$$

В силу требования перенормируемости взаимодействие полей описывается стандартным вкладом $i e \bar{\psi} \hat{A} \psi$ в действие КЭД.

В качестве дефекта мы будем рассматривать материальную плоскость $x_{3}=0$. В этом случае в дираковской части действия нашей модели

$$
S(\bar{\psi}, \psi)=\int \bar{\psi}(x)\left(i \hat{\partial}-m+\Omega\left(x_{3}\right)\right) \psi(x) d x
$$

взаимодействие спинорного поля с плоскостью описывается матрицей $\Omega\left(x_{3}\right)=Q \delta\left(x_{3}\right)$. Так как $\Omega\left(x_{3}\right)$ и $\delta\left(x_{3}\right)$ имеют размерность массы, то матрица $Q$ безразмерна. В силу симметрии нашей системы она должна быть инвариантна относительно преобразований подгруппы группы Пуанкаре, не меняющих координаты $x_{3}$. В наиболее общем случае матрица $Q$ может быть представлена в виде

$$
Q=q_{1} I+q_{2} \gamma_{5}+q_{3} \gamma_{3}+q_{4} \gamma_{5} \gamma_{3}
$$

где $I$ - единичная $(4 \times 4)$-матрица, а $\gamma_{3}, \gamma_{5}=i \gamma_{0} \gamma_{1} \gamma_{2} \gamma_{3}-$ матрицы Дирака, для которых мы используем следующие представления:

$$
\begin{aligned}
\gamma_{0}=\left(\begin{array}{rrrr}
1 & 0 & 0 & 0 \\
0 & 1 & 0 & 0 \\
0 & 0 & -1 & 0 \\
0 & 0 & 0 & -1
\end{array}\right), & \gamma_{1}=\left(\begin{array}{rrrrr}
0 & 0 & 0 & 1 \\
0 & 0 & 1 & 0 \\
0 & -1 & 0 & 0 \\
-1 & 0 & 0 & 0
\end{array}\right), \\
\gamma_{2}=\left(\begin{array}{rrrr}
0 & 0 & 0 & -i \\
0 & 0 & i & 0 \\
0 & i & 0 & 0 \\
-i & 0 & 0 & 0
\end{array}\right), & \gamma_{3}=\left(\begin{array}{rrrr}
0 & 0 & 1 & 0 \\
0 & 0 & 0 & -1 \\
-1 & 0 & 0 & 0 \\
0 & 1 & 0 & 0
\end{array}\right) .
\end{aligned}
$$

Движение спинорной частицы в поле дефекта $\Omega\left(x_{3}\right)$ описывается уравнением Дирака

$$
\left(i \hat{\partial}-m+\Omega\left(x_{3}\right)\right) \psi(x)=0
$$


Оно является одним из уравнений Эйлера-Лагранжа для действия (1), которое получается при его вариационном дифференцировании по $\bar{\psi}(x)$. В результате дифференцирования $(1)$ по $\psi(x)$ получается второе уравнение:

$$
\left(\partial_{\mu} \bar{\psi}(x)\right) \gamma^{\mu}+\bar{\psi}(x)\left(m-\Omega\left(x_{3}\right)\right)=0
$$

Нетрудно убедиться, что $\bar{\psi}(x)=\psi^{*}(x) \gamma_{0}$, если $\gamma_{0} \Omega^{+}\left(x_{3}\right)=\Omega\left(x_{3}\right) \gamma_{0}$.

В дальнейшем мы будем использовать для пространственных частей векторов пространства-времени обозначения

$$
\bar{x}=\left(x_{0}, x_{1}, x_{2}\right), \quad \bar{p} \bar{x}=p_{0} x_{0}-p_{1} x_{1}-p_{2} x_{2} .
$$

Функция $\psi(x)=\psi\left(\bar{x}, x_{3}\right)$, удовлетворяющая уравнению $(3)$, не может быть непрерывной при $x_{3}=0$ и не может иметь в этой точке сингулярности дельтаобразного типа. Представим ее в виде $\psi(x)=\psi_{s}(x)+\psi_{a}(x)$, где

$$
\psi_{s}(x)=\frac{1}{2}\left(\psi\left(\bar{x}, x_{3}\right)+\psi\left(\bar{x},-x_{3}\right)\right), \quad \psi_{a}(x)=\frac{1}{2}\left(\psi\left(\bar{x}, x_{3}\right)-\psi\left(\bar{x},-x_{3}\right)\right) .
$$

Мы предполагаем, что пределы справа и слева $x_{3} \rightarrow \pm 0$ у функции $\psi(x)$ существуют. Тогда функция $\psi_{s}(x)$ в точке $x_{3}=0$ непрерывна, а пределы $x_{3} \rightarrow \pm 0$ функции $\psi_{a}(x)$ справа и слева различаются знаками. Проинтегрируем по $x_{3}$ в пределах от $-a$ до $a, a>0$, уравнение $(3)$ и вычислим затем предел $a \rightarrow 0$. В результате мы получим

$$
\begin{gathered}
-i \gamma_{3} \psi_{a}(\bar{x})+Q \lim _{a \rightarrow 0} \int_{-a}^{a} \delta\left(x_{3}\right) \psi(x) d x_{3}=0, \\
\psi_{a}(\bar{x})=\lim _{x_{3} \rightarrow+0} \psi(x)-\lim _{x_{3} \rightarrow-0} \psi(x)=2 \lim _{x_{3} \rightarrow+0} \psi_{a}(x) .
\end{gathered}
$$

Оставшийся в (4) интеграл непосредственно из определения дельта-функции не вычисляется, так как функция имеет разрыв при $x_{3}=0$. Поэтому заменим в (4) дельта-функцию ее регуляризованным выражением

$$
\delta_{\lambda}\left(x_{3}\right)=\sqrt{\frac{\lambda}{2 \pi}} e^{-\lambda x_{3}^{2} / 2}
$$

и вычислим интеграл в пределе $\lambda \rightarrow \infty$. Мы имеем

$$
\begin{aligned}
& \int_{-a}^{a} \delta_{\lambda}\left(x_{3}\right) \psi\left(\bar{x}, x_{3}\right) d x_{3}=\sqrt{\frac{\lambda}{2 \pi}} \int_{-a}^{a} e^{-\lambda x_{3}^{2} / 2} \psi(x) d x_{3}= \\
& \quad=\sqrt{\frac{\lambda}{2 \pi}} \int_{-a}^{a} e^{-\lambda x_{3}^{2} / 2} \psi_{s}(x) d x_{3}=\sqrt{\frac{1}{2 \pi}} \int_{-a \sqrt{\lambda}}^{a \sqrt{\lambda}} e^{-x_{3}^{2} / 2} \psi_{s}\left(\bar{x}, \frac{x_{3}}{\sqrt{\lambda}}\right) d x_{3}
\end{aligned}
$$


Таким образом, получаем

$$
\begin{gathered}
\int_{-a}^{a} \delta\left(x_{3}\right) \psi\left(\bar{x}, x_{3}\right) d x_{3}=\lim _{\lambda \rightarrow \infty} \int_{-a}^{a} \delta_{\lambda}\left(x_{3}\right) \psi\left(\bar{x}, x_{3}\right) d x_{3}=\psi_{s}(\bar{x}) \\
\psi_{s}(\bar{x})=\left.\frac{1}{2}(\psi(x)+\psi(-x))\right|_{x_{3}=0} .
\end{gathered}
$$

В итоге наша задача может быть сформулирована следующим образом. При $x_{3} \neq 0$ поле $\psi(x)$ удовлетворяет уравнению Дирака

$$
(i \hat{\partial}-m) \psi(x)=0
$$

а при $x_{3}=0$ выполняется соотношение

$$
-i \gamma_{3} \psi_{a}(\bar{x})+Q \psi_{s}(\bar{x})=0
$$

Общее решение уравнения (5) при $x_{3}>0$ можно представить в виде

$$
\psi(x)=\frac{1}{(2 \pi)^{3}} \int e^{i \bar{p} \bar{x}} \psi_{+}\left(\bar{p}, x_{3}\right) d \bar{p} .
$$

Тогда из $(5)$ следует, что $\psi_{+}\left(\bar{p}, x_{3}\right)$ удовлетворяет уравнению

$$
\left(i \gamma_{3} \partial_{3}+\hat{\bar{p}}+m\right) \psi_{+}\left(\bar{p}, x_{3}\right)=0 .
$$

Его общее решение имеет вид

$$
\psi_{+}\left(\bar{p}, x_{3}\right)=U\left(\bar{p}, x_{3}\right) \chi_{+}(\bar{p}), \quad U\left(\bar{p}, x_{3}\right)=e^{i \gamma_{3}(\hat{p}+m) x_{3}},
$$

где $\chi_{+}(\bar{p})-$ произвольный спинор, зависящий только от $\bar{p}$.

Аналогично при $x_{3}<0$ получаем

$$
\psi(x)=\frac{1}{(2 \pi)^{3}} \int e^{i \bar{p} \bar{x}} \psi_{-}\left(\bar{p}, x_{3}\right) d \bar{p}, \quad \psi_{-}\left(\bar{p}, x_{3}\right)=U\left(\bar{p}, x_{3}\right) \chi_{-}(\bar{p}) .
$$

Из (6)-(8) получаем соотношение

$$
-i \gamma_{3}\left(\chi_{+}-\chi_{-}\right)+Q\left(\chi_{+}+\chi_{-}\right)=0
$$

из которого следует

$$
\chi_{-}=S \chi_{+}, \quad S=\left(i \gamma_{3}+Q\right)^{-1}\left(i \gamma_{3}-Q\right)
$$

Введя обозначения

$$
\kappa(\bar{p})=\sqrt{\bar{p}^{2}-m^{2}}, \quad P^{ \pm}(\bar{p})=\frac{1}{2}\left(1 \mp \frac{\gamma_{3}(\hat{\bar{p}}+m)}{\kappa(\bar{p})}\right),
$$


мы можем представить $U\left(\bar{p}, x_{3}\right)$ в виде

$$
\begin{aligned}
U\left(\bar{p}, x_{3}\right) & =e^{i \gamma_{3}(\hat{\bar{p}}+m) x_{3}}=\cos \left(\kappa(\bar{p}) x_{3}\right)+i \sin \left(\kappa(\bar{p}) x_{3}\right) \frac{\gamma_{3}(\hat{\bar{p}}+m)}{\kappa(\bar{p})}= \\
& =e^{i \kappa(\bar{p}) x_{3}} P^{-}(\bar{p})+e^{-i \kappa(\bar{p}) x_{3}} P^{+}(\bar{p}) .
\end{aligned}
$$

Матрицы $P^{ \pm}(\bar{p})$ являются проекторами:

$$
\begin{gathered}
P^{ \pm}(\bar{p}) P^{ \pm}(\bar{p})=P^{ \pm}(\bar{p}), \quad P^{+}(\bar{p})+P^{-}(\bar{p})=1, \\
P^{+}(\bar{p}) P^{-}(\bar{p})=P^{-}(\bar{p}) P^{+}(\bar{p})=0 .
\end{gathered}
$$

Каждый из них имеет два собственных двумерных подпространства $L^{+}$и $L^{-}$, которые представляются в виде линейной комбинаций двух взаимно ортогональных собственных векторов $v_{1}^{ \pm}, v_{2}^{ \pm}$:

$$
\begin{gathered}
L^{ \pm}\left(a_{1}^{ \pm}, a_{2}^{ \pm}\right)=a_{1}^{ \pm} v_{1}^{ \pm}+a_{2}^{ \pm} v_{2}^{ \pm}, \\
P^{ \pm}(\bar{p}) L^{ \pm}\left(a_{1}^{ \pm}, a_{2}^{ \pm}\right)=L^{ \pm}\left(a_{1}^{ \pm}, a_{2}^{ \pm}\right), \quad P^{ \pm}(\bar{p}) L^{\mp}\left(a_{1}^{\mp}, a_{2}^{\mp}\right)=0 .
\end{gathered}
$$

Здесь $a_{i}^{ \pm}, i=1,2,-$ комплексные параметры, а $v_{i}^{ \pm}, i=1,2,-$ спиноры, которые мы выбираем в виде

$$
\begin{gathered}
v_{1}^{-}=n_{1}^{-1}\left(-h_{1}, h_{2}^{-}, 1, h_{3}^{-}\right), \quad v_{2}^{-}=n_{2}^{-1}\left(h_{1}, h_{2}^{+}, 1, h_{3}^{+}\right), \\
v_{1}^{+}=n_{2}^{-1}\left(-h_{1}, h_{2}^{+}, 1,-h_{3}^{+}\right), \quad v_{2}^{+}=n_{1}^{-1}\left(h_{1}, h_{2}^{-}, 1,-h_{3}^{-}\right), \\
n_{1}=2 \sqrt{\frac{p_{0} h_{2}^{-}}{p_{1}+i p_{2}}}, \quad n_{2}=2 \sqrt{\frac{p_{0} h_{2}^{+}}{p_{1}+i p_{2}}}, \quad h_{1}=\frac{\sqrt{p_{0}^{2}-m^{2}}}{m+p_{0}}, \\
h_{2}^{ \pm}=\frac{p_{0}^{2}-m^{2} \pm \sqrt{p_{0}^{2}-m^{2}} \kappa(\bar{p})}{\left(m+p_{0}\right)\left(p_{1}-i p_{2}\right)}, \quad h_{3}^{ \pm}=\frac{\kappa(\bar{p}) \pm \sqrt{p_{0}^{2}-m^{2}}}{p_{1}-i p_{2}} .
\end{gathered}
$$

Эти спиноры являются собственными для оператора спиральности

$$
\sigma(\vec{p})=\frac{i}{2|\vec{p}|}(\vec{p} \vec{s}), \quad \vec{s}=\left(\gamma_{2} \gamma_{3},-\gamma_{1} \gamma_{3}, \gamma_{1} \gamma_{2}\right)
$$

Для них выполняются соотношения

$$
\left.\sigma(\vec{p})\right|_{p_{3}= \pm \kappa(\bar{p})} v_{1}^{ \pm}=-\frac{1}{2} v_{1}^{ \pm},\left.\quad \sigma(\vec{p})\right|_{p_{3}= \pm \kappa(\bar{p})} v_{2}^{ \pm}=\frac{1}{2} v_{2}^{ \pm} .
$$

Представим спиноры $\chi_{ \pm}(\bar{p})$ в виде

$$
\chi_{ \pm}(\bar{p})=b_{ \pm}^{1}(\bar{p}) v_{1}^{-}(\bar{p})+b_{ \pm}^{2}(\bar{p}) v_{2}^{-}(\bar{p})+c_{ \pm}^{1}(\bar{p}) v_{1}^{+}(\bar{p})+c_{ \pm}^{2}(\bar{p}) v_{2}^{+}(\bar{p}) .
$$

Нетрудно убедиться, что справедливо равенство

$$
\chi_{ \pm}^{*}(\bar{p}) \gamma_{0} \gamma_{3} \chi_{ \pm}(\bar{p})=\bar{\chi}_{ \pm}(\bar{p}) \gamma_{3} \chi_{ \pm}(\bar{p})=\frac{\kappa(\bar{p})}{p_{0}}\left(\left|c_{ \pm}^{1}\right|^{2}+\left|c_{ \pm}^{2}\right|^{2}-\left|b_{ \pm}^{1}\right|^{2}-\left|b_{ \pm}^{2}\right|^{2}\right) .
$$


Таким образом, если $p_{0}>0, \kappa(\bar{p})>0$, то амплитуды $c_{ \pm}^{i}$ описывают движение частиц в направлении $x_{3}>0$, а $b_{ \pm}^{i}-$ в противоположном направлении.

Основываясь на уравнении (3), мы исследуем процесс рассеяния спинорных частиц на плоскости дефекта, а также свойства локализованных в окрестности дефекта связанных состояний.

\section{3. РАССЕЯНИЕ ЧАСТИЦ НА ПЛОСКОСТИ $x_{3}=0$}

Решение уравнений Дирака (3) описывает движение свободных частиц при $x_{3} \neq 0$, если $\kappa(\bar{p})>0$. Это условие всегда выполняется для поля Дирака $\psi(x)$ в случае однородного и изотропного пространства, и ток, определяемый как

$$
j^{\mu}(x)=\bar{\psi}(x) \gamma^{\mu} \psi(x)=\psi^{*}(x) \gamma^{0} \gamma^{\mu} \psi(x),
$$

удовлетворяет уравнению непрерывности $\partial_{\mu} j^{\mu}(x)=0$, вследствие чего полный ток $I$ вдоль оси $x_{3}$

$$
I=\int j_{3}(x) d \bar{x}=-\int j^{3}(x) d \bar{x}
$$

не зависит от координаты $x_{3}$. Для нашей модели определим токи $j_{ \pm}^{\mu}(x)$ в полупространствах $x_{3}>0$ и $x_{3}<0$ :

$$
j_{ \pm}^{\mu}(x)=\bar{\psi}_{ \pm}(x) \gamma^{\mu} \psi_{ \pm}(x)=\psi_{ \pm}^{*}(x) \gamma^{0} \gamma^{\mu} \psi_{ \pm}(x)
$$

Соответствующие им полные токи

$$
I_{ \pm}=-\int j_{ \pm}^{3}(x) d \bar{x}
$$

не зависят от координаты $x_{3}$. Выразим токи $I_{ \pm}$через введенные нами в $(7),(8)$ спиноры $\chi_{ \pm}(\bar{p})$. Вследствие (11) имеем

$$
I_{ \pm}=\int \bar{\kappa}_{ \pm}(\bar{p}) \gamma_{3} \kappa(\bar{p}) d \bar{p}=\int \frac{\kappa(\bar{p})}{p_{0}}\left(\left|c_{ \pm}^{1}\right|^{2}+\left|c_{ \pm}^{2}\right|^{2}-\left|b_{ \pm}^{1}\right|^{2}-\left|b_{ \pm}^{2}\right|^{2}\right) d \bar{p} .
$$

Таким образом, если $p_{0}>0$, то амплитуды $c_{ \pm}^{1}, c_{ \pm}^{2}$ описывают частицы, движущиеся в положительном направлении оси $x_{3}$, a $b_{ \pm}^{1}, b_{ \pm}^{2}-$ в отрицательном.

Рассмотрим процесс, в котором первоначальный поток частиц $I_{\text {in }}$ с импульсом $\vec{p}=\left(p_{1}, p_{2}, \kappa(\bar{p})\right)$ движется к плоскости $x_{3}=0$ из области $x_{3}<0$. Его характеристики определяются амплитудами $c_{-}^{1}, c_{-}^{2}$. В области $x_{3}<0$ также имеется поток отраженных частиц, заданный амплитудами $b_{-}^{1}, b_{-}^{2}$. В области $x_{3}>0$ имеются только частицы, движущиеся в положительном направлении. Их поток описывается амплитудами $c_{+}^{1}, c_{+}^{2}$. Таким образом, для рассматриваемого процесса коэффициенты отражения $K_{\mathrm{r}}$ и прохождения $K_{\mathrm{tr}}$ имеют вид

$$
K_{\mathrm{r}}=\frac{\left|b_{-}^{1}\right|^{2}+\left|b_{-}^{2}\right|^{2}}{\left|c_{-}^{1}\right|^{2}+\left|c_{-}^{2}\right|^{2}}, \quad K_{\mathrm{tr}}=\frac{\left|c_{+}^{1}\right|^{2}+\left|c_{+}^{2}\right|^{2}}{\left|c_{-}^{1}\right|^{2}+\left|c_{-}^{2}\right|^{2}} .
$$

Амплитуды $c_{-}^{1}, b_{-}^{1}, c_{+}^{1}$ соответствуют частицам с отрицательной спиральностью, a $c_{-}^{2}, b_{-}^{2}, c_{+}^{2}-$ с положительной спиральностью. Для рассматриваемого нами процесса рассеяния амплитуды $c_{-}^{1}, c_{-}^{2}$ считаются заданными, а $b_{-}^{1}, b_{-}^{2}, c_{+}^{1}, c_{+}^{2}$ находятся 
из уравнения $(9)$, связывающего $\chi_{+}$с $\chi_{-}$, и из уравнений

$$
b_{+}^{1}=0, \quad b_{+}^{2}=0 .
$$

Проведем анализ уравнения (9), необходимый для решения поставленной задачи. Непосредственно из этого уравнения следует, что если

$$
S^{+} \gamma_{0} \gamma_{3} S=\gamma_{0} \gamma_{3}
$$

то $\bar{\chi}_{-}(\bar{p}) \gamma_{3} \chi_{-}(\bar{p})=\bar{\chi}_{+}(\bar{p}) \gamma_{3} \chi_{+}(\bar{p})$ и $I_{+}=I_{-}$. Таким образом, соотношение $(13)$ представляет собой условие отсутствия поглощения и излучения частиц плоскостью $x_{3}=0$, в результате чего полный ток $I$ оказывается не зависящим от координаты $x_{3}$.

Так как определитель матрицы $\gamma_{0} \gamma_{3}$ равен единице, то из (13) получаем, что $|\operatorname{det} S|=1$. Из свойств симметрии рассматриваемой системы следует, что $S$ имеет вид

$$
S=s_{1} I+s_{2} \gamma_{5}+s_{3} \gamma_{3}+s_{4} \gamma_{5} \gamma_{3}
$$

Нетрудно убедиться, что для матрицы вида (14) справедливы равенства

$$
\begin{gathered}
S^{-1}=\left(s_{1} I-s_{2} \gamma_{5}-s_{3} \gamma_{3}-s_{4} \gamma_{5} \gamma_{3}\right)\left(s_{1}^{2}-s_{2}^{2}+s_{3}^{2}-s_{4}^{2}\right)^{-1}, \\
\operatorname{det} S=\left(s_{1}^{2}-s_{2}^{2}+s_{3}^{2}-s_{4}^{2}\right)^{2} .
\end{gathered}
$$

Так как условие (13) можно записать в виде $S^{-1}=\gamma_{0} \gamma_{3} S^{+} \gamma_{0} \gamma_{3}$, то вследствие эрмитовости $I, \gamma_{5}, \gamma_{5} \gamma_{3}$, антиэрмитовости $\gamma_{3}$ и свойств коммутации этих матриц с $\gamma_{0}, \gamma_{3}$ мы имеем

$$
S^{-1}=s_{1}^{*} I+s_{2}^{*} \gamma_{5}+s_{3}^{*} \gamma_{3}-s_{4}^{*} \gamma_{5} \gamma_{3}
$$

Воспользовавшись тем, что $|\operatorname{det} S|=1$, представим $\operatorname{det} S$ в виде $\operatorname{det} S=e^{2 i \eta}$, где $0 \leqslant \eta<\pi$. Сравнивая (17) с (15), (16), получаем

$$
\begin{array}{rrr}
s_{1}^{*}=e^{-i \eta} s_{1}, & s_{2}^{*}=-e^{-i \eta} s_{2}, & s_{3}^{*}=-e^{-i \eta} s_{3}, \quad s_{4}^{*}=e^{-i \eta} s_{4}, \\
s_{1}= \pm e^{i \eta / 2}\left|s_{1}\right|, & s_{2}= \pm i e^{i \eta / 2}\left|s_{2}\right|, & s_{3}= \pm i e^{i \eta / 2}\left|s_{3}\right|, \quad s_{4}= \pm e^{i \eta / 2}\left|s_{4}\right| .
\end{array}
$$

Таким образом, если выполняется соотношение (13), то, выбрав параметры $\varsigma_{1}, \varsigma_{2}, \varsigma_{3}$, $\varsigma_{4}$ вещественными, матрицу $S$ можно представить в виде $S=e^{i \varphi} \mathcal{S}$, где $0 \leqslant \varphi<2 \pi$ и

$$
\begin{gathered}
\mathcal{S}=\varsigma_{1} I+i \varsigma_{2} \gamma_{5}+i \varsigma_{3} \gamma_{3}+\varsigma_{4} \gamma_{5} \gamma_{3}, \\
\varsigma_{1}^{2}+\varsigma_{2}^{2}-\varsigma_{3}^{2}-\varsigma_{4}^{2}=1 .
\end{gathered}
$$

Из соотношения (9) можно выразить $Q$ через $S$ :

$$
Q=i \gamma_{3}(1-S)(1+S)^{-1} \text {. }
$$

Отсюда с учетом (18) получаем выражения для коэффициентов $q_{i}, 1 \leqslant i \leqslant 4$, в формуле (2) в терминах параметров $\varsigma_{i}$ :

$$
q_{1}=-\frac{\varsigma_{3}}{1+\varsigma_{1}}, \quad q_{2}=-\frac{i \varsigma_{4}}{1+\varsigma_{1}}, \quad q_{3}=0, \quad q_{4}=-\frac{\varsigma_{2}}{1+\varsigma_{1}} .
$$

6 Теоретическая и математическая физика, т. 184, № 3, 2015 г. 
Из этих соотношений следует, что $q_{2}$ мнимо, а $q_{1}, q_{4}$ вещественны. Соотношения (18) и (19) позволяют выразить $\varsigma_{i}$ через $q_{i}$ :

$$
\begin{aligned}
\varsigma_{1}=-1+\frac{2}{1-q_{1}^{2}+q_{2}^{2}+q_{4}^{2}}, & \varsigma_{2}=-\frac{2 q_{4}}{1-q_{1}^{2}+q_{2}^{2}+q_{4}^{2}}, \\
\varsigma_{3}=-\frac{2 q_{1}}{1-q_{1}^{2}+q_{2}^{2}+q_{4}^{2}}, & \varsigma_{4}=\frac{2 i q_{2}}{1-q_{1}^{2}+q_{2}^{2}+q_{4}^{2}} .
\end{aligned}
$$

Подставив в уравнение (9) выражение для матрицы $S$ в виде (18), а $\chi_{ \pm}$в виде (10), получим линейные уравнения для амплитуд $b_{ \pm}^{1}, b_{ \pm}^{2}, c_{+}^{1}, c_{+}^{2}$. Их решение с учетом (12) позволяет найти потоки отраженных и прошедших частиц различных поляризаций. Введем обозначения

$$
\begin{gathered}
c_{11}=c_{-}^{1 *} c_{-}^{1}, \quad c_{22}=c_{-}^{2 *} c_{-}^{2}, \quad c_{s}=c_{-}^{2 *} c_{-}^{1}+c_{-}^{1 *} c_{-}^{2}, \quad c_{a}=c_{-}^{2 *} c_{-}^{1}-c_{-}^{1 *} c_{-}^{2}, \\
g=p_{0}\left(p_{0}^{2}-m^{2}\right)\left(\left(\left(\varsigma_{2}^{2}-\varsigma_{3}^{2}\right) m^{2}+\left(\varsigma_{1}^{2}+\varsigma_{2}^{2}\right) \kappa^{2}\right)^{2}+4 \varsigma_{1}^{2} \varsigma_{3}^{2} m^{2} \kappa^{2}\right) .
\end{gathered}
$$

Поток отраженных частиц с положительной спиральностью оказывается равным

$$
\left|b_{-}^{2}\right|^{2}=\frac{c_{11} r_{1}^{+}+c_{22} r_{2}^{+}+c_{s} r_{3}^{+}+i c_{a} r_{4}^{+}}{g}
$$

где

$$
\begin{aligned}
r_{1}^{+}= & -m^{2}\left(p_{1}^{2}+p_{2}^{2}\right) \kappa\left(\left(\varsigma_{1} \varsigma_{3}+\varsigma_{2} \varsigma_{4}\right)^{2} \kappa^{2}+\left(\varsigma_{2}^{2}-\varsigma_{3}^{2}\right)^{2} m^{2}\right), \\
r_{2}^{+}= & -\kappa^{3}\left(\left(\left(\varsigma_{2}^{2}-\varsigma_{3}^{2}\right) m p_{0}+\left(\varsigma_{2} \varsigma_{3}-\varsigma_{1} \varsigma_{4}\right) \kappa \sqrt{p_{0}^{2}-m^{2}}\right)^{2}+\right. \\
& \left.+\left(\left(\varsigma_{1} \varsigma_{3}+\varsigma_{2} \varsigma_{4}\right) p_{0} \kappa+\left(\varsigma_{1} \varsigma_{2}-\varsigma_{3} \varsigma_{4}\right) m \sqrt{p_{0}^{2}-m^{2}}\right)^{2}\right), \\
r_{3}^{+}= & -\kappa^{2} m \sqrt{p_{1}^{2}+p_{2}^{2}}\left(p_{0}\left(\left(\varsigma_{2}^{2}-\varsigma_{3}^{2}\right)^{2} m^{2}+\left(\varsigma_{1} \varsigma_{3}+\varsigma_{2} \varsigma_{4}\right)^{2} \kappa^{2}\right)+\right. \\
& \left.+\varsigma_{2} \varsigma_{3} m \kappa \sqrt{p_{0}^{2}-m^{2}}\right), \\
r_{4}^{+}= & -\kappa^{2} m \sqrt{p_{0}^{2}-m^{2}} \sqrt{p_{1}^{2}+p_{2}^{2}}\left(\left(\varsigma_{2} \varsigma_{3}-\varsigma_{1} \varsigma_{4}\right)\left(\varsigma_{1} \varsigma_{3}+\varsigma_{2} \varsigma_{4}\right) \kappa^{2}-\right. \\
& \left.-\left(\varsigma_{2}^{2}-\varsigma_{3}^{2}\right)\left(\varsigma_{1} \varsigma_{2}-\varsigma_{3} \varsigma_{4}\right) m^{2}\right) .
\end{aligned}
$$

Поток отраженных частиц с отрицательной спиральностью описывается следующим образом:

$$
\left|b_{-}^{1}\right|^{2}=\frac{c_{11} r_{1}^{-}+c_{22} r_{2}^{-}+c_{s} r_{3}^{-}+i c_{a} r_{4}^{-}}{g},
$$

где $r_{2}^{-}=r_{1}^{+}, r_{4}^{-}=-r_{4}^{+}$,

$$
\begin{aligned}
r_{1}^{-}= & -\kappa^{3}\left(\left(\left(\varsigma_{2}^{2}-\varsigma_{3}^{2}\right) m p_{0}-\left(\varsigma_{2} \varsigma_{3}-\varsigma_{1} \varsigma_{4}\right) \kappa \sqrt{p_{0}^{2}-m^{2}}\right)^{2}+\right. \\
& \left.+\left(\left(\varsigma_{1} \varsigma_{3}+\varsigma_{2} \varsigma_{4}\right) p_{0} \kappa-\left(\varsigma_{1} \varsigma_{2}-\varsigma_{3} \varsigma_{4}\right) m \sqrt{p_{0}^{2}-m^{2}}\right)^{2}\right), \\
r_{3}^{-}= & \kappa^{2} m \sqrt{p_{1}^{2}+p_{2}^{2}}\left(p_{0}\left(\left(\varsigma_{2}^{2}-\varsigma_{3}^{2}\right)^{2} m^{2}+\left(\varsigma_{1} \varsigma_{3}+\varsigma_{2} \varsigma_{4}\right)^{2} \kappa^{2}\right)-\varsigma_{2} \varsigma_{3} m \kappa \sqrt{p_{0}^{2}-m^{2}}\right) .
\end{aligned}
$$


Поток пролетевших частиц с положительной спиральностью представляется в виде

$$
\left|c_{+}^{2}\right|^{2}=\frac{c_{11} t_{1}^{+}+c_{22} t_{2}^{+}+c_{s} t_{3}^{+}+i c_{a} t_{4}^{+}}{g},
$$

где

$$
\begin{aligned}
t_{1}^{+} & =\kappa^{3}\left(\left(\varsigma_{3} m \sqrt{p_{0}^{2}-m^{2}}+\varsigma_{2} p_{0} \kappa\right)^{2}+\varsigma_{1}^{2}\left(p_{0}^{2}-m^{2}\right) \kappa^{2}\right), \\
t_{2}^{+} & =\varsigma_{2}^{2} \kappa^{3} m^{2}\left(p_{1}^{2}+p_{2}^{2}\right), \\
t_{3}^{+} & =-\kappa^{3} \varsigma_{2} m \sqrt{p_{1}^{2}+p_{2}^{2}}\left(\varsigma_{3} m \sqrt{p_{0}^{2}-m^{2}}+\varsigma_{2} p_{0} \kappa\right), \\
t_{4}^{+} & =\kappa^{4} \varsigma_{1} \varsigma_{2} m \sqrt{p_{0}^{2}-m^{2}} \sqrt{p_{1}^{2}+p_{2}^{2}} .
\end{aligned}
$$

Для потока пролетевших частиц с отрицательной спиральностью мы получаем

$$
\left|c_{+}^{1}\right|^{2}=\frac{c_{11} t_{1}^{-}+c_{22} t_{2}^{-}+c_{s} t_{3}^{-}+i c_{a} t_{4}^{-}}{g}
$$

где $t_{1}^{-}=t_{2}^{+}, t_{4}^{-}=-t_{4}^{+}$,

$$
\begin{aligned}
& t_{2}^{-}=\kappa^{3}\left(\left(\varsigma_{3} m \sqrt{p_{0}^{2}-m^{2}}-\varsigma_{2} p_{0} \kappa\right)^{2}+\varsigma_{1}^{2}\left(p_{0}^{2}-m^{2}\right) \kappa^{2}\right), \\
& t_{3}^{-}=-\kappa^{3} \varsigma_{2} m \sqrt{p_{1}^{2}+p_{2}^{2}}\left(\varsigma_{3} m \sqrt{p_{0}^{2}-m^{2}}-\varsigma_{2} p_{0} \kappa\right) .
\end{aligned}
$$

Для частного случая движения частиц ортогонально плоскости дефекта $\left(p_{1}=0\right.$, $\left.p_{2}=0\right)$ удобно использовать обозначение

$$
g_{1}=p_{0}\left(\left(\varsigma_{2}^{2} p_{0}^{2}-\varsigma_{3}^{2} m^{2}+\varsigma_{1}^{2}\left(p_{0}^{2}-m^{2}\right)\right)^{2}+4 \varsigma_{1}^{2} \varsigma_{3}^{2} m^{2}\left(p_{0}^{2}-m^{2}\right)\right) .
$$

В этом случае потоки отраженных и пролетевших частиц с положительной и отрицательной спиральностью оказываются равными

$$
\begin{aligned}
\left|b_{-}^{2}\right|^{2} & =\frac{c_{22} r_{2}^{+}}{g_{1}}, \\
r_{2}^{+} & =-\sqrt{p_{0}^{2}-m^{2}}\left(\left(\varsigma_{2} p_{0}-\varsigma_{3} m\right)^{2}+\varsigma_{1}^{2}\left(p_{0}^{2}-m^{2}\right)\right)\left(\left(\varsigma_{3} p_{0}+\varsigma_{2} m\right)^{2}+\varsigma_{4}^{2}\left(p_{0}^{2}-m^{2}\right)\right), \\
\left|b_{-}^{1}\right|^{2} & =\frac{c_{11} r_{1}^{-}}{g_{1}}, \\
r_{1}^{-} & =-\sqrt{p_{0}^{2}-m^{2}}\left(\left(\varsigma_{2} p_{0}+\varsigma_{3} m\right)^{2}+\varsigma_{1}^{2}\left(p_{0}^{2}-m^{2}\right)\right)\left(\left(\varsigma_{3} p_{0}-\varsigma_{2} m\right)^{2}+\varsigma_{4}^{2}\left(p_{0}^{2}-m^{2}\right)\right), \\
\left|c_{+}^{2}\right|^{2} & =\frac{c_{11} t_{1}^{+}}{g_{1}}, \\
t_{1}^{+} & =\left(p_{0}^{2}-m^{2}\right)^{3 / 2}\left(\left(\varsigma_{2} p_{0}+\varsigma_{3} m\right)^{2}+\varsigma_{1}^{2}\left(p_{0}^{2}-m^{2}\right)\right), \\
\left|c_{+}^{1}\right|^{2} & =\frac{c_{22} t_{2}^{-}}{g_{1}}, \\
t_{2}^{-} & =\left(p_{0}^{2}-m^{2}\right)^{3 / 2}\left(\left(\varsigma_{2} p_{0}-\varsigma_{3} m\right)^{2}+\varsigma_{1}^{2}\left(p_{0}^{2}-m^{2}\right)\right) .
\end{aligned}
$$




\section{4. СВЯЗАННЫЕ СОСТОЯНИЯ}

Локализованные в окрестности $x_{3}=0$ состояния получаются, если $\bar{p}^{2}-m^{2}<0$ и $\kappa(\bar{p})$ является мнимым. По определению мы считаем, что $\kappa(\bar{p})=i|\kappa(\bar{p})|$. В этом случае мы получаем условия отсутствия экспоненциального роста $\psi(x)$ при $x_{3} \rightarrow$ $\pm \infty$ :

$$
P^{+} \chi_{+}(\bar{p})=0, \quad P^{-} \chi_{-}(\bar{p})=0 .
$$

Таким образом, $c_{+}^{j}=0, b_{-}^{j}=0, j=1,2$, и

$$
\chi_{+}(\bar{p})=v_{1}^{-} b_{+}^{1}+v_{2}^{-} b_{+}^{2}, \quad \chi_{-}(\bar{p})=v_{1}^{+} c_{-}^{1}+v_{2}^{+} c_{-}^{2},
$$

и в силу (9) амплитуды $b_{+}^{1}, b_{+}^{2}$ удовлетворяют соотношениям

$$
v_{1}^{+*} \gamma_{0} \gamma_{3} S \chi_{+}(\bar{p})=\frac{\kappa(\bar{p})}{p_{0}} c_{+}^{1}=0, \quad v_{2}^{+*} \gamma_{0} \gamma_{3} S \chi_{+}(\bar{p})=\frac{\kappa(\bar{p})}{p_{0}} c_{+}^{2}=0,
$$

из которых получаем систему уравнений

$$
\alpha^{-} b_{+}^{1}+\beta b_{+}^{2}=0, \quad \beta b_{+}^{1}+\alpha^{+} b_{+}^{2}=0 .
$$

Здесь мы использовали обозначения

$$
\begin{aligned}
\alpha^{ \pm} & =\frac{i \sqrt{p_{0}^{2}-m^{2}}\left(\varsigma_{3} m-\varsigma_{1} \lambda\right) \pm \varsigma_{2} p_{0} \lambda}{\sqrt{p_{0}^{2}-m^{2}}}, \\
\beta & =\frac{i \varsigma_{2} m \sqrt{p_{0}^{2}\left(p_{0}^{2}-m^{2}+\lambda^{2}\right)}}{p_{0} \sqrt{p_{0}^{2}-m^{2}}}
\end{aligned}
$$

и $\lambda=|\kappa(\bar{p})|$. Вследствие (9) амплитуды $c_{-}^{1}, c_{-}^{2}$, определяющие $\chi_{-}(\bar{p})$ в $(20)$, выражаются в терминах $b^{1}+, b_{+}^{2}$ :

$$
\begin{aligned}
& c_{-}^{1}=\frac{\varsigma_{3} m \sqrt{p_{0}^{2}\left(p_{0}^{2}-m^{2}+\lambda^{2}\right)}}{p_{0} \lambda \sqrt{p_{0}^{2}-m^{2}}} b_{+}^{1}+\frac{\sqrt{p_{0}^{2}-m^{2}}\left(\varsigma_{2} m+\varsigma_{4} \lambda\right)-i \varsigma_{3} p_{0} \lambda}{\lambda \sqrt{p_{0}^{2}-m^{2}}} b_{+}^{2}, \\
& c_{-}^{2}=\frac{\sqrt{p_{0}^{2}-m^{2}}\left(\varsigma_{2} m+\varsigma_{4} \lambda\right)+i \varsigma_{3} p_{0} \lambda}{\lambda \sqrt{p_{0}^{2}-m^{2}}} b_{+}^{1}+\frac{\varsigma_{3} m \sqrt{p_{0}^{2}\left(p_{0}^{2}-m^{2}+\lambda^{2}\right)}}{p_{0} \lambda \sqrt{p_{0}^{2}-m^{2}}} b_{+}^{2} .
\end{aligned}
$$

Из (21) следует обращение в нуль двух линейных комбинаций этих уравнений:

$$
\left(\alpha^{+} \alpha^{-}-\beta^{2}\right) b_{+}^{1}=0, \quad\left(\alpha^{+} \alpha^{-}-\beta^{2}\right) b_{+}^{2}=0 .
$$

Таким образом, нетривиальное решение $b_{+}^{1}, b_{+}^{2}$ системы (21) мы можем записать следующим образом:

$$
\begin{array}{r}
b_{+}^{j}=\delta(f(\bar{p})) \varphi_{j}(\bar{p}), \quad j=1,2, \\
f(\bar{p})=\left(\alpha^{+} \alpha^{-}-\beta^{2}\right)=\left(\varsigma_{3} m-\varsigma_{1} \lambda\right)^{2}-\varsigma_{2}^{2}\left(m^{2}-\lambda^{2}\right) .
\end{array}
$$


Taк как

$$
f(\bar{p})=\left(\varsigma_{1}^{2}+\varsigma_{2}^{2}\right)\left(\lambda-\lambda^{+}\right)\left(\lambda-\lambda^{-}\right), \quad \lambda^{ \pm}=\frac{\varsigma_{1} \varsigma_{3} \pm \varsigma_{2}^{2} \sqrt{1+\varsigma_{4}^{2}}}{\varsigma_{1}^{2}+\varsigma_{2}^{2}} m,
$$

и $\lambda$ по определению положительно, то

$$
\delta(f(\bar{p})) \varphi_{i}(\bar{p})=\theta(\lambda) \delta(f(\bar{p})) \varphi_{i}(\bar{p})=\theta\left(\lambda^{+}\right) \delta\left(\lambda-\lambda^{+}\right) \varphi_{i}^{+}(\bar{p})+\theta\left(\lambda^{-}\right) \delta\left(\lambda-\lambda^{-}\right) \varphi_{i}^{-}(\bar{p}) .
$$

В силу уравнений $(21)$ функции $\varphi_{2}^{ \pm}(\bar{p})$ выражаются через $\varphi_{1}^{ \pm}(\bar{p})$ :

$$
\varphi_{2}^{ \pm}(\bar{p})=\gamma^{ \pm}\left(p_{0}\right) \varphi_{1}^{ \pm}(\bar{p})
$$

где

$$
\gamma^{ \pm}\left(p_{0}\right)=-\left.\frac{\alpha^{-}}{\beta}\right|_{\lambda_{=\lambda^{ \pm}}}=-\left.\frac{\beta}{\alpha^{+}}\right|_{\lambda_{=\lambda^{ \pm}}}=-\frac{p_{0}\left(\sqrt{p_{0}^{2}-m^{2}}\left(\varsigma_{3} m-\varsigma_{1} \lambda^{ \pm}\right)+i \varsigma_{2} p_{0} \lambda^{ \pm}\right)}{\left.\varsigma_{2} m \sqrt{p_{0}^{2}\left(p_{0}^{2}-m^{2}+\lambda^{ \pm 2}\right.}\right)} .
$$

Мы имеем

$$
\begin{gathered}
\theta\left(\lambda^{ \pm}\right) \delta\left(\lambda^{ \pm 2}-\lambda^{2}\right)=\theta\left(\lambda^{ \pm}\right) \delta\left(2 \lambda^{ \pm}\left(\lambda^{ \pm}-\lambda\right)\right)=\frac{\theta\left(\lambda^{ \pm}\right)}{2 \lambda^{ \pm}} \delta\left(\lambda^{ \pm}-\lambda\right), \\
\lambda^{ \pm 2}-\lambda^{2}=p_{0}^{2}-p_{1}^{2}-p_{2}^{2}-m^{2}+\lambda^{ \pm 2}=\bar{p}^{2}-\xi^{ \pm} m^{2}, \quad \xi^{ \pm}=1-\lambda^{ \pm 2} .
\end{gathered}
$$

Так как

$$
\xi^{ \pm}=1-\left(\frac{\varsigma_{1} \varsigma_{3} \pm \varsigma_{2}^{2} \sqrt{1+\varsigma_{4}^{2}}}{\varsigma_{1}^{2}+\varsigma_{2}^{2}}\right)^{2}
$$

то коэффициент $\xi^{ \pm}$при соответствующем значении параметров $\varsigma_{i}$ может быть положительным, отрицательным и равным нулю. Так, например, $\xi^{+}=0$ при $\varsigma_{1}=\varsigma_{3}$, $\varsigma_{2}=1, \varsigma_{4}=0$, а $\xi^{-}=0$ при $\varsigma_{1}=-\varsigma_{3}, \varsigma_{2}=1, \varsigma_{4}=0$. Если $\varsigma_{2}=0, \varsigma_{1}>\sqrt{1+\varsigma_{3}^{2}}$, $\varsigma_{4}= \pm \sqrt{\varsigma_{1}^{2}-1-\varsigma_{3}^{2}}$, то $\xi^{ \pm}>0$, и $\xi^{+}<0$, если $\varsigma_{1}=1, \varsigma_{2}=\varsigma_{3}>1, \varsigma_{4}=0$. Случай $\xi^{-}<0$ не реализуется.

Закон дисперсии $\bar{p}^{2}=\xi^{ \pm} m^{2}$ описывает свободные частицы с эффективной массой $m \sqrt{\xi^{ \pm}}<m$ в $(2+1)$-мерном пространстве-времени с двумя пространственными и одной временно́й координатами, если $\xi^{ \pm}>0$. Если $\xi^{+}=0$ или $\xi^{-}=0$, то соответствующие частицы безмассовые. Движением таких частиц объясняются многие эффекты в графене. Если $\xi^{+}<0$, то возникают состояния, для которых $p_{1}^{2}+p_{2}^{2}>p_{0}^{2}>0$, и соответствующий им ток, параллельный плоскости дефекта, не может быть равен нулю. Такие состояния подобны сверхпроводящим.

\section{5. ЗАКЛЮЧЕНИЕ}

В настоящей работе построена модель взаимодействия фермионного поля с материальной плоскостью в рамках подхода Симанзика. Функционал действия модели включает обычное спинорное действие Дирака и дополнительный вклад дефекта, сосредоточенный в занятой им области пространства. Действие содержит три параметра, характеризующие свойства материала плоскости. В модели проведены расчеты характеристик процессов рассеяния дираковской частицы на плоскости дефекта, а также исследованы свойства локализованных вблизи нее состояний. 
Модель и полученные на ее основе результаты можно использовать для теоретического описания процессов взаимодействия электронов, позитронов и нейтронов с двумерными материалами (графен, тонкие пленки, напыления, резкие границы твердого тела). Простые модификации модели позволяют учесть эффекты воздействия внешних электромагнитных полей.

Применение подхода Симанзика в КЭД дает возможность построить модель для широкого класса квантовых макроскопических явлений в двухмерных материалах. Такая модель может оказаться полезной для исследования эффекта Холла, плазмон-поляритонных эффектов, нанофотоники, свойств топологических изоляторов, тонких пленок, напылений и резких границ [16].

Благодарности. Работа выполнена при поддержке Санкт-Петербургского государственного университета (грант № 11.38.660.2013).

\section{Список литературы}

[1] Н. Н. Боголюбов, Д. В. Ширков, Квантовые поля, Наука, М., 1993; К. Ициксон, Ж.-Б. Зюбер, Квантовая теория поля, Мир, М., 1984.

[2] H. B. G. Casimir, Proc. K. Ned. Akad. Wet., 51 (1948), 793-795.

[3] U. Mohideen, A. Roy, Phys. Rev. Lett., 81:21 (1998), 4549-4552, arXiv: physics/9805038; A. Roy, C.-Y. Lin, U. Mohideen, Phys. Rev. D, 60:11 (1999), 111101, 5 pp., arXiv: quant-ph/9906062.

[4] B. W. Harris, F. Chen, U. Mohideen, Phys. Rev. A, 62:5 (2000), 052109, 5 pp., arXiv: quant-ph/0005088.

[5] G. Bressi, G. Carugno, R. Onofrio, G. Ruoso, Phys. Rev. Lett., 88:4 (2002), 041804, 4 pp., arXiv: quant-ph/0203002.

[6] M. Bordag, G. L. Klimchitskaya, U. Mohideen, V. M. Mostepanenko, Advances in the Casimir Effect, The International Series of Monographs on Physics, 145, Oxford Univ. Press, Oxford, 2009; G. L. Klimchitskaya, U. Mohideen, V. M. Mostepanenko, Rev. Modern Phys., 81:4 (2009), 1827-1885, arXiv: 0902.4022.

[7] K. A. Milton, J. Phys. A: Math. Gen., 37:38 (2004), R209-R277.

[8] K. Symanzik, Nucl. Phys. B, 190:1 (1981), 1-44.

[9] V.N. Markov, Yu. M. Pis'mak, J. Phys. A: Math. Gen., 39:21 (2006), 6525-6532, arXiv: hep-th/0606058; Casimir effect for thin films from imperfect material, arXiv: hep-th/0505218.

[10] V. N. Marachevsky, Yu. M. Pis'mak, Phys. Rev. D, 81:6 (2010), 065005, 6 pp., arXiv: 0907.1985.

[11] Д. Ю. Письмак, Ю. М. Письмак, ТМФ, 175:3 (2013), 442-454; 169:1 (2011), 69-78; D. Yu. Pis'mak, Yu. M. Pis'mak, ЭЧАЯ, 44:3 (2013), 879-898.

[12] D. Yu. Pis'mak, Yu. M. Pis'mak, F. J. Wegner, Phys. Rev. E, 92:1 (2015), 013204, 7 pp., arXiv: 1406.1598.

[13] K. I. Kondo, T. Ebihara, T. Iizuka, E. Tanaka, Nucl. Phys. B, 434:1 (1995), 85-108, arXiv: hep-ph/9404361; T. Appelquist, M. J. Bowick, D. Karabali, L. C. R. Wijewardhana, Phys. Rev. D, 33:12 (1986), 3774-3776.

[14] A. N. Grigorenko, M. Polini, K.S. Novoselov, Nature Photonics, 6:11 (2012), 749-758, arXiv: 1301.4241; A. H. Castro Neto, F. Guinea, N. M. R. Peres, K.S. Novoselov, A. K. Geim, Rev. Modern Phys., 81:1 (2009), 109-162; I. V. Fialkovsky, D. V. Vassilevich, J. Phys. A: Math. Theor., 42:44 (2009), 442001, 6 pp.; W.-K. Tse, A. H. MacDonald, Phys. Rev. B, 84:20 (2011), 205327, 13 pp., arXiv: 1108.3858; L. Chen, S. Wan, Phys. Rev. B, 84 (2011), 075149, 6 pp.; J. González, JHEP, 07 (2013), 175, arXiv: 1211.3905; 
V. N. Kotov, B. Uchoa, V. M. Pereira, F. Guinea, A. H. Castro Neto, Rev. Modern Phys., 84:3 (2012), 1067-1125, arXiv: 1012.3484; I. V. Fialkovsky, V. N. Marachevsky, D. V. Vassilevich, Phys. Rev. B, 84:3 (2011), 035446, 10 pp., arXiv: 1102.1757; G. L. Klimchitskaya, V. M. Mostepanenko, B.E. Sernelius, Phys. Rev. B, 89:12 (2014), 125407, 9 pp., arXiv: 1401.6886; G. L. Klimchitskaya, V. M. Mostepanenko, Phys. Rev. A, 89:5 (2014), 052512, 7 pp., arXiv: 1404.7057; J. E. Moore, Nature, 464:728 (2010), 194-198.

[15] I. V. Fialkovsky, V. N. Markov, Yu. M. Pis'mak, J. Phys. A: Math. Gen., 39:21 (2006), 6357-6363; Internat. J. Modern Phys. A, 21:12 (2006), 2601-2616, arXiv: hep-th/0311236.

[16] G. Bracco, B. Holst (eds.), Surface Science Techniques, Springer Series in Surface Science, 51, Springer, Berlin, 2013; M. L. Brongersma, P. G. Kik (ed.), Surface Plasmon Nanophotonics, Springer Series in Optical Sciences, 131, Springer, Berlin, 2007; S. A. Maier, Plasmonics: Fundamental and Applications, Springer, Berlin, 2007. 